\title{
Comparison of International Federation of cervical pathology and colposcopy nomenclature (2011) and Swede score for assessment of cervical lesions
}

Bindiya Gupta ( $\sim$ dr_bindiya_gupta@yahoo.co.in )

UCMS \& GTB Hospital https://orcid.org/0000-0002-2555-9607

Rashmi Shreya

UCMS \& GTB Hospital

Shalini Rajaram

AllMS

Anshuja Singla

UCMS \& GTB Hospital

Sandhya Jain

UCMS \& GTB Hospital

Bhavya Prasad

UCMS \& GTB Hospital

\section{Research Article}

Keywords: Cervical cancer, Colposcopy, Cervical intraepithelial neoplasia, colposcopy nomenclature, Swede score, IFCPC nomenclature

Posted Date: June 1st, 2021

DOl: https://doi.org/10.21203/rs.3.rs-169936/v1

License: (c) (i) This work is licensed under a Creative Commons Attribution 4.0 International License.

Read Full License 


\section{Title page}

\section{Title: Comparison of International Federation of cervical pathology and colposcopy nomenclature (2011) and Swede score for assessment of cervical lesions}

\section{Authors:}

Bindiya Gupta MD ${ }^{1}$, Rashmi Shreya MD $^{2}$, Shalini Rajaram MD ${ }^{3}$, Anshuja Singhla DGo DNB ${ }^{4}$, Sandhya Jain MS $^{5}$, Bhavya Prasad $\mathrm{MS}^{5}$

${ }^{1}$ Associate Professor, Department of Obstetrics and Gynecology, UCMS \& GTB Hospital, Delhi

2Consultant Max Saket, Former Senior Resident, Department of Obstetrics and Gynecology, UCMS \& GTB

Hospital, Delhi

${ }^{3}$ Professor, Gynecologic Oncology, Department of Obstetrics and Gynecology, AlIMS, Rishikesh

${ }^{4}$ Associate Professor, Department of Obstetrics and Gynecology, UCMS \& GTB Hospital, Delhi

${ }^{5}$ Associate Professor, Department of Obstetrics and Gynecology, UCMS \& GTB Hospital, Delhi

${ }^{6}$ Senior resident, Department of Obstetrics and Gynecology, UCMS \& GTB Hospital, Delhi

Running title: Standardised terminology for colposcopic findings

There is no potential conflict of interest

There is no funding

Word Count: 2805

Number of figures: 2

Number of tables: 2

Corresponding author

Dr Bindiya Gupta

Associate Professor

Obstetrics and Gynecology, UCMS \& GTB Hospital, Delhi

Email: dr_bindiya_gupta@yahoo.co.in

Phone :+91-9810719002

\section{Abstract}

Purpose: Standardization of colposcopic evaluation is important as it is observer dependent.The aim of the study was to compare the strength of association of colposcopic findings by International Federation of Colposcopy and Cervical Pathology (IFCPC) nomenclature and Swede score.

Methods: In the prospective study, 150 women aged 30 to 60 years with abnormal screening results underwent colposcopy, the findings were evaluated using both IFCPC and Swede score and biopsy was taken from abnormal areas. Performances by both the methods were calculated taking biopsy as gold standard. 
Results: Nineteen $\geq$ CIN2 lesions were detected. The sensitivity, specificity, positive predictive value and negative predictive value for detecting $\geq$ CIN2 lesion by IFCPC scoring was $63.6 \%, 96.0 \%, 78.7 \%, 91.9 \%$. Using Swede score, sensitivity, specificity, positive predictive value and negative predictive value at cut off of 8 were $42.4,95 \%, 96.6 \%$ and $81.8 \%$ while at cut-off of 5 , these were $96 \%, 88.3 \%, 76.7 \%$ and $93.6 \%$. The agreement between IFCPC major lesion and Swede score $\geq 8$ for $\geq$ CIN2 was $0.626(p<0.01)$.

Conclusion: Swede score is an easier and more objective method for colposcopic evaluation and a score $>8$ can be applied to the screen and treat programme.

Keywords: Cervical cancer, Colposcopy, Cervical intraepithelial neoplasia, colposcopy nomenclature, Swede score, IFCPC nomenclature

\section{Comparison of International Federation of cervical pathology and colposcopy nomenclature (2011) and}

\section{Swede Score for assessment of cervical lesions}

\section{INTRODUCTION}

Cervical cancer is the leading cause of mortality and morbidity amongst women and is the fourth most common form of cancer in women with 18.1 million new cases of cervical cancer worldwide and 9.6 million deaths according to GLOBOCAN 2018 [1]. It remains a major public health problem in developing countries where more than $85 \%$ of these cases and deaths occur [2]. Cervical cancer is a preventable disease as it can be diagnosed in its precancerous stage and if detected early and treated optimally, it results in a high cure rate. Hence, screening for cervical cancer using visual inspection tests, human papilloma virus (HPV) testing and or Pap smear is of paramount importance $[3,4]$. Screening is followed by colposcopy and directed biopsy that can diagnose cervical precancer and cancer in early stages.

Various colposcopic scoring and grading systems are available. Reid and Scalzi proposed the Reid's Colposcopic Index (RCl) with a sensitivity of $89 \%$ for any lesion but $56 \%$ for a high-grade lesion. The specificity for low-grade lesions was $57.5 \%$ which increased to $92.9 \%$ for high-grade lesion. This scoring system was objective and widely used but had a pitfall of not including the lesion size $[5,6]$.

The international colposcopic terminology, the 2011 International Federation of cervical pathology and colposcopy nomenclature (IFCPC) nomenclature has been proposed for several years; however, the evaluation studies are few in number [7]. Li Y et al showed that the reproducibility of TZ and the predictive value of a few signs still need to be discussed [8].

Although colposcopy by IFCPC nomenclature 2011 provides standardized and uniform interpretations, it is still considered a subjective assessment and is highly observer dependent for a trained colposcopist. In a retrospective cohort study using IFCPC terminology the diagnosis was overestimated in $19.9 \%$ and underestimated in $19.1 \%$. The authors concluded that although the common findings were classified reasonably, and some signs were highly reliable the reproducibility of transformation zone types and the implication of lesion size needed further discussion [9]. 
Swede score includes the lesion size and it is an objective scoring system [10]. A study by Bowring J et al showed that the score had a good positive predictive value, there was no major learning curve and the trainees showed comparable results like their trained colleagues [11]. The present study was designed to compare the Swede score with IFCPC terminology and determine whether it has an equal diagnostic accuracy.

\section{MATERIAL AND METHODS}

It was a prospective study, carried out in the gynecology department from July 2016 to September 2017. Taking $42.5 \%$ sensitivity of Swede score and $15 \%$ prevalence of $\geq$ CIN2 lesions, at $20 \%$ precision, sample size was calculated as 150. A written informed consent was taken from all participants and ethical clearance was obtained from the institutional ethical committee.

One hundred and fifty screen positive women with abnormal screening results (positive Pap smear, positive VIA or visual inspection with Lugol iodine) aged 30 to 59 years were included. Exclusion criteria included presence of an obvious growth, previous procedures on the cervix, acute pelvic inflammatory disease and pregnant women. Demographic variables, menstrual, obstetric history, past and family history of medical disorders and socio economic status were noted. A note was made of symptoms, previous screening tests, previous history of treatment or sexually transmitted disease, age at first intercourse and number of sexual partners.

Socioeconomic class was divided into upper, upper middle, middle class, lower middle, and lower according to the modified BG Prasad scale, 2016 based on per capita monthly income [12].

Colposcopy was performed by authors 1 (BG ), 3 (SR) and 4 (SJ). Author 3 (SR) has 20 years of performing colposcopies and is a trainer for colposcopy courses by International Federation of Colposcopy and Cervical Pathology (IFCPC) and International agency of research on cancer (IARC). Author 1 (BG) and 4 (SJ) have an experience of 10 years of performing colposcopy and author $1(B G)$ is an accredited colposcopist by IFCPC and IARC. Colposcopy was performed using Frontline * video colposcope.

Adequate colposcopy was defined as all 4 quadrants of cervix visible. Green filter was used to evaluate the vessels. Acetic acid 5\% (weight/volume) was applied and observation was noted after 1 minute of application and visualization of the cervix was done under magnification. The transformation zone was identified, and the type of transformation zone was described as type 1, 2, or 3. The margins and surface configuration of the lesion were graded followed by assessment after Lugol's iodine staining. The findings were reported using International Federation of Cervical Pathology and Colposcopy Terminology, 2011. The lesions were graded as minor, major, suspicious for invasion or miscellaneous depending on the acetowhiteness, vasculature, margins and signs like inner border sign and rag sign [7]. Swede score was calculated taking into account acetowhiteness, margins, vessel pattern, lesion size and Lugol's staining. Each variable was graded as 0,1,2, and total score was calculated (maximum 10) [10]. Depending on a computer based random number table either the swede score or the IFCPC criteria was calculated first to avoid bias. Cervical biopsies were taken using a Tischler forceps in cases where lesion was graded as major on IFCPC terminology or calculated Swede score was more than or equal to 5 and sent for histopathology. Cases in which biopsies were not taken were considered benign or negative.

Statistical Analysis 
Sensitivity, specificity, positive and negative predictive value were analysed for the two scoring systems taking with histopathological diagnosis as gold standard ( $\geq$ CIN2 as cut off). The level of agreement between scores and biopsy were demonstrated by Kappa statistics taking ( $\geq \mathrm{CIN} 2$ ) as cut off). ROC analysis was carried out to determine cut off score for better sensitivity and specificity values. To study agreement between IFCPC terminology and Swede score the Mc Nemar's statistics were used. Data was analysed using statistical product service solutions (SPSS) software IBM version 22

\section{RESULTS}

A total of 150 women referred for colposcopy were recruited in this study. The mean age was $38.17 \pm 8.6$ years. The mean parity was $3.13 \pm 1.37$. Mean age at marriage and at first intercourse was 18 years; $80.7 \%$ women were from low socioeconomic status coming with chief complaints of vaginal discharge (42.7\%) and abnormal uterine bleeding (37.3\%). Baseline variables are mentioned in Table-1.

Out of 60 biopsies taken, 29 (19.3\%) were chronic cervicitis, 11 (7.3\%) were cervical intraepithelial neoplasia-1 (CIN -1), 8 (5.3\%) CIN -2, 6 (4\%) were CIN 3, adeno carcinoma $3(2 \%)$, invasive squamous cell carcinoma $2(1.3 \%)$ and tuberculosis $1(0.65 \%)$.

Colposcopy was adequate in all cases. Squamocolumnar junction was visible completely/partially in $91.3 \%$ and was not visible in $8.7 \%$. Transformation zone (TZ) was T1 in 100 cases (67.3\%), T2TZ in 33 cases (22\%) and type T3TZ in 16 cases (10.7\%). In accordance with IFCPC 2011, major lesion was seen in 132 (88.7\%) cases while minor lesion was seen in 18 (11.3\%) cases. Using IFCPC 2011 terminology, sensitivity, specificity, positive and negative predictive value for major lesion was $68.4 \%, 90.0 \%, 76.5 \%$, $85.7 \%$ respectively while for minor lesion was $47.2 \%, 65.0 \%, 52.2 \%, 73.4 \%$ respectively.

The distribution of Swede score variables is shown in Table-2. The mean Swede score for $\leq$ CIN1 was 3 (range: 1-6) while for $\geq$ CIN2 was 7 (range 5-10). Sensitivity, specificity, positive and negative predictive value using a cut off of swede score 5 was 100\%, 40.2\%, 44.2\%,100\% while for swede score 8 and more sensitivity, specificity, positive and negative predictive value was $57.9 \%, 87.5 \%, 68.8 \%, 81.4 \%$.

Both IFCPC terminology and Swede score showed high diagnostic accuracy for $\geq$ CIN2 disease. The receiver operative characteristics curve (ROC curve) is shown in Figure 1 and 2. The area under the curve for IFCPC terminology and Swede score was 0.96 and 0.88 respectively $(p=0.75)$. Taking a cut of off $\geq$ CIN2 disease, measurement of agreement between IFCPC terminology major lesion was $r=0.601$ while that of minor lesion was $r=0.4012$. Measurement of agreement between Swede score more than or equal to 8 to biopsy positive ( $\geq$ CIN2 was 0.622 while that with minor lesion was 0.30 . The agreement between IFCPC major lesion and Swede score $>8$ for $\geq$ CIN2 was $r=0.626(p<0.01)$.

\section{DISCUSSION}

The present study demonstrated that both the IFCPC terminology and Swede score showed high diagnostic accuracy for $\geq$ CIN2 disease and are comparable with moderate degree of agreement for diagnosis of $\geq$ CIN2 disease $(r=0.626)$.

Various colposcopic scoring system are available like the IFCPC nomenclature, Reid's colposcopic index, Swede scoring system etc. $[5,7,9,10]$. 
Kushwah S et al in their study reported that at a cut off of Swede score of 8 the specificity was 100\% (92.32100) and sensitivity was $36.84 \%$ for CIN2+ lesions [13]. Another study by Bowring J et al showed that Swede scores of 8 or more has a sensitivity, specificity, and positive and negative predictive values of 38\%, 95\%, 83\%, and $70 \%$, respectively, for lesions for CIN 2+ disease [11]. Study by Ranga $R$ et al showed that at a higher cut off of 8 the the sensitivity was lower as compared to a cut off of 5 at $42.42 \%$ versus $100 \%$ respectively [14]. The specificity was much higher at a higher cut off of $8(100 \%$ versus $88.37 \%)$ respectively. In the present study also showed that at a cut off of 8 , sensitivity, specificity, and positive and negative predictive values of $57.9 \%$, $87.5 \%, 68.8 \%, 81.4 \%$ which is almost similar to study by Bowring J et al. The sensitivity was higher at a lower cut off of 5 while specificity for $\geq$ CIN2 disease was much higher at a swede score cut off 8 in the present study which is similar to the study by Ranga et al.

Studies have compared Swede score with Reid's colposcopic index showing significant high degree of correlation between the two scores $0.937(p<.001)$ [14]. Kushwah $S$ et al also showed that there was an excellent correlation between the Reid's score and Swede score, the correlation coefficient $\left(R^{2}\right)$ was 0.924 [13]. Only one study has compared the IFCPC nomenclature and Swede score. Li Y et al showed that in trained colposcopists, the sensitivity for IFCPC classification was $63.64 \%$ and specificity was $96 \%$ for a cut off for highgrade squamous intraepithelial lesion (HSIL+). The agreement with cervical pathology was 0.64 . They concluded that significant differences existed between the IFCPC classification and the Swede score $(\chi 2=96.46, P<$ 0.001) using the Mc Nemar's statistics. However, this was a retrospective study and the Swede Score was calculated from colposcopy photographs, thus having a bias. The main strength is that it is the first prospective study to compare the two scoring systems. In the present study also using IFCPC 2011 terminology, for major lesion the sensitivity and specificity was similar at 68.4\%, 90.0\% respectively. However, the degree of agreement between two scores showed a moderate agreement $(r=0.626)$ at a Swede score cut off of 8 for high grade disease.

Colposcopy by IFCPC terminology is subjective and highly dependent on observer's evaluation. Swede score is more objective and can be used flexibly depending on the scoring. The lower threshold 5 with high sensitivity can be used for screening, whereas the higher threshold 8 and above with high specificity and above can be used for screen and treat (to minimize overtreatment in see and treat program). As the study by Bowring J et al had shown that the learning curve of Swede score is less, it can hence be effectively used for interpretation of results and can be used by nurses and paramedics after appropriate training.

The only limitation in the study was that the colposcopist was not blinded to the screening test thus creating bias.

\section{CONCLUSION}

To conclude, Swede score is an easier and more objective method for colposcopic evaluation in screen positive patients. Using a higher cut off of 8 the specificity increases for high grade disease while at a cut off of 5 the sensitivity is higher. It can be easily used as an effective alternative to the IFCPC terminology. A higher cut off can be applied to the screen and treat programme while a lower cut off can be used for screening only.

Acknowledgements: nil 


\section{The Statement of Ethics:}

\section{Subjects (or their parents or guardians) have given their written informed consent.}

The study protocol has been approved by the research institute's committee on human research.

"The authors have no ethical conflicts to disclose."

"The authors have no conflicts of interest to declare."

Funding : nil

\section{$\underline{\text { Author contribution }}$}

Protocol/project development: Shalini Rajaram, Bindiya Gupta, Rashmi Shriya

Collection of data and management: Bindiya Gupta, Rashmi Shriya, Bhavya Prasad, Sandhya Jain

Analysis and Interpretation of data: Bindiya Gupta, Rashmi Shriya, Anshuja Singhla,

Writing/ editing of manuscript: Bindiya Gupta, Shalini Rajaram, Rashmi Shriya, Bhavya Prasad

Critical revision: Shalini Rajaram, Bindiya Gupta, Anshuja Singhla, Sandhya Jain

\section{References}

1. World Health Organization (WHO). Cervical Cancer Estimate Incidence, Mortality and Prevalence Worldwide in 2018. Available at:

http://www.iarc.fr/en/mediacentre/iarcnews/2018/gco_globocan2018.php. Accessed on 15th September 2020.

2. Dikshit R, Gupta PC, Ramasundarahettige C, et al (2012) Cancer mortality in India: A nationally representative survey. Lancet 379:1807-1816.

3. Denny L, Quinn M, Sankaranarayanan R. Screening for cervical cancer in developing countries Available at: http://www.who.int/immunization/sage/Dennycervical_cancer.pdf. Accessed on 17.1.2018.

4. Lee H, Kang Y, Ju W (2016) Cervical Cancer Screening in Developing Countries: Using Visual Inspection Methods. Clin J Oncol Nurs 20:79-83.

5. Reids R, Stanhope CR, Herschman BR, Crum CP, Agronow SJ (1984) Genital warts and cervical cancer. A colposcopic index for differentiating subclinical papilloma viral infection from cervical intraepithelial neoplasia. Am J Obstet Gynecol 149:815-823.

6. Reid R, Scalzi P (1985) Genital warts and cervical cancer an improved colposcopic index for differentiating benign papillomaviral infections from high-grade cervical intraepithelial neoplasia. Am J Obstet Gynecol 153:611-618

7. Bornstein J, Bentley J, Bösze P et al (2012) 2011 colposcopic terminology of the International Federation for Cervical Pathology and Colposcopy. Obstet Gynecol. 120:166-172.

8. Li Y, Duan X, Sui L, et al (2017) Closer to a Uniform Language in Colposcopy: Study on the Potential Application of 2011International Federation for Cervical Pathology and Colposcopy Termi nology in Clinical Practice. Biomed Res Int :8984516. 
9. Li Y, Zhang H, Zheng R, Xie F, Sui L (2015) Agreement between colposcopic diagnosis with 2011 international terminology of colposcopy and cervical pathology in cervical lesions. Zhonghua Fu Chan Ke Za Zhi 50:361- 366.

10. Strander B, Ellström-Andersson A, Franzén S, Milsom I, Rådberg T (2005) The performance of a new scoring system for colposcopy in detecting high-grade dysplasia in the uterine cervix. Acta Obstet Gynecol Scand 84:1013-1017.

11. Bowring J, Strander B, Young M, Evans H, Walker P (2010) The Swede score: evaluation of a scoring system designed to improve the predictive value of colposcopy. J Low Genit Tract Dis 14:301- 305.

12. Kharinar MR, WadgaveU, Shimpi PV (2016) Updates BG Prasad socioeconomic classification for 2016. J Indian Assoc Public Health Dent 14: 469- 470.

13. Kushwah $\mathrm{S}$, Kushwah B Correlation of Two Colposcopic Indices for Predicting Premalignant Lesions of Cervix . J Midlife Health 8:118- 123.

14. Ranga R, Rai S, Kumari A, Mathur S, Kriplani A, Mahey R (2017) A Comparison of the Strength of Association of Reid Colposcopic Index and Swede Score With Cervical Histology. J Low Genit Tract Dis. 21:55- 58.

\section{Figure legends}

Figure 1: ROC curve for IFCPC terminology

Figure 2: ROC curve for Swede score 
Table-1: Baseline variables

\begin{tabular}{|c|c|}
\hline Variable & $n=150$ \\
\hline Age (years)* & $38.17 \pm 8.6$ \\
\hline Parity* & $3.13+1.374$ \\
\hline Age at marriage (years)* & $18.84 \pm 2.060$ \\
\hline Age at first intercourse* & $18.93 \pm 1.929$ \\
\hline Age at menarche* & $13.26+1.736$ \\
\hline \multicolumn{2}{|l|}{ Religion } \\
\hline Hindu & $115(76.7 \%)$ \\
\hline Muslims & $34(22.7 \%)$ \\
\hline Christians & $1(0.7 \%)$ \\
\hline \multicolumn{2}{|l|}{ Socioeconomic status } \\
\hline Middle class & $29(19.3 \%)$ \\
\hline Low & $121(80.7 \%)$ \\
\hline \multicolumn{2}{|l|}{ Chief complaints } \\
\hline Vaginal discharge & $64(42.7 \%)$ \\
\hline Abnormal uterine bleeding & $56(37.3 \%)$ \\
\hline Pain abdomen & $14(9.3 \%)$ \\
\hline Post coital bleeding & $15(10 \%)$ \\
\hline More than one complaint & $35(23.3 \%)$ \\
\hline \multicolumn{2}{|l|}{ Contraceptive use } \\
\hline Yes & $124(83.3 \%)$ \\
\hline No & $26(16.7 \%)$ \\
\hline $\begin{array}{l}\text { Previous History of } \\
\text { - Abnormal screening tests }\left(\mathrm{Pap} / \mathrm{VIA}^{\star \star}\right) \\
\text { - Previous procedures of the cervix } \\
\text { - previous sexually transmitted disease }\end{array}$ & $\begin{array}{l}6(4 \%) \\
1(0.6 \%) \\
2(1.3 \%)\end{array}$ \\
\hline Number of previous sexual partners & 1 \\
\hline
\end{tabular}

*Mean (SD)

** VIA: Visual inspection with acetic acid 
Table 2: Distribution of Swede score variables

\begin{tabular}{|c|c|}
\hline Variable & $\begin{array}{l}\mathrm{N}=150 \\
\mathrm{~N}(\text { Percentage })\end{array}$ \\
\hline \multicolumn{2}{|l|}{ Acetouptake } \\
\hline 0 Zero/transparent & $99(66 \%)$ \\
\hline 1 Shady/milky & $25(16.7 \%)$ \\
\hline 2 Distinct, opaque white & $26(17.3 \%)$ \\
\hline \multicolumn{2}{|l|}{ Margins/ surface } \\
\hline 0 Diffuse & $97(64.6 \%)$ \\
\hline 1 Sharp but irregular, jagged, satellites & $42(28 \%)$ \\
\hline 2 Sharp, difference in surface, cuffing & $11(7.3 \%)$ \\
\hline Vessels & \\
\hline 0 Fine, regular & $102(68 \%)$ \\
\hline 1 Absent & $34(22.6 \%)$ \\
\hline 2 Coarse, atypical & $14(9.3 \%)$ \\
\hline Lesion size & \\
\hline 0 less than $5 \mathrm{~mm}$ & $88(58.7 \%)$ \\
\hline $15-15 \mathrm{~mm} / 2$ quadrants & $42(28 \%)$ \\
\hline $2>15 \mathrm{~mm} />2$ quadrants, endocervical & $20(13.3 \%)$ \\
\hline \multicolumn{2}{|l|}{ Iodine staining } \\
\hline 0 Brown & $88(58.7 \%)$ \\
\hline 1 Faintly/ patchy yellow & $39(26 \%)$ \\
\hline 2 Distinct yellow & $23(15.3 \%)$ \\
\hline
\end{tabular}


Figure 1: ROC curve for IFCPC terminology

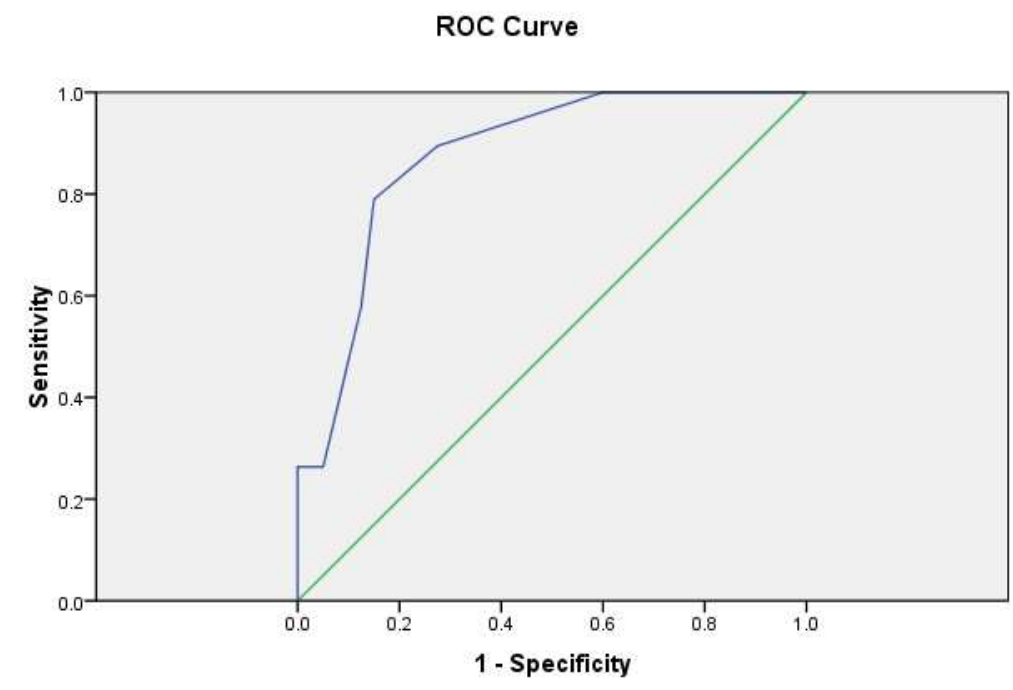

Diagonal segments are produced by ties.

Figure 2: ROC curve for Swede score

ROC Curve

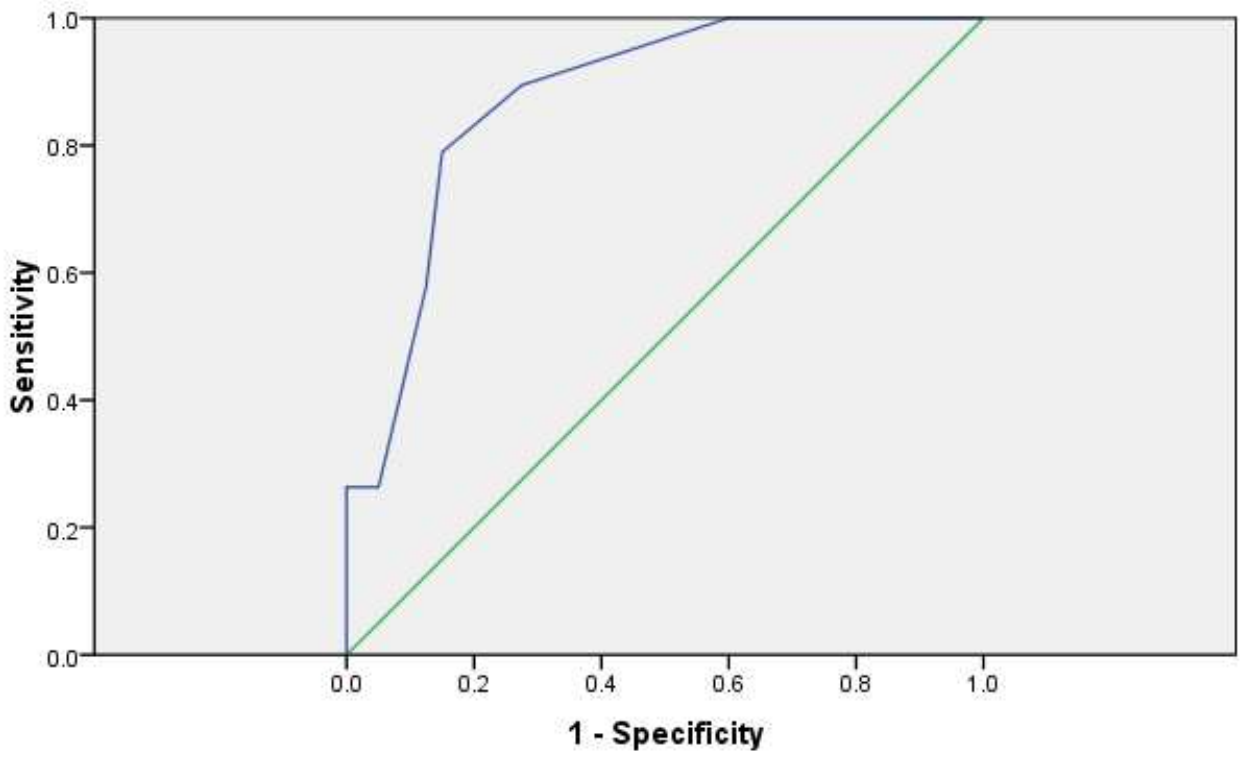

Diagonal segments are produced by ties. 
